\title{
SATISFAÇÃO E LEALDADE DISCENTE NAS UNIVERSIDADES PÚBLICAS BRASILEIRAS
}

\section{Satisfaction and student loyalty in Brazilian public universities}

Roberta Hoffmann

E-mail: betahm@gmail.com

MBA em Gestão de Projetos pela Universidade Estácio de Sá; Graduada em Engenharia de Produção pela Universidade Federal de Pelotas; Especialista de Performance de Processos na Multinacional JTI. https://orcid.org/ 0000-0003-4476-0936

Éverton Cavalheiro

E-mail: eacavalheiro@hotmail.com

Doutor e Mestre pela Universidade Federal de Santa Maria; Professor na Universidade Federal de

Pelotas.

https://orcid.org/0000-0002-6767-3889

Leonardo Betemps Kontz

E-mail: leonardobetemps@gmail.com

Doutor em Engenharia de Produção pela Universidade do Vale do Rio dos Sinos; Mestre em Ciências

Sociais pela Universidade Federal de Pelotas; Professor no Instituto Federal Sul-rio-grandense Endereço para contato: Rua Gonçalves Chaves, 3218, centro, 96015-560, Pelotas, Rio Grande do Sul, Brasil.

http://orcid.org/0000-0003-2052-375X

Alejandro Martins

E-mail: aljmartins@gmail.com

Doutor e Mestre em Engenharia de Produção pela Universidade Federal de Santa Catarina;

Professor na Universidade Federal de Pelotas.

http://orcid.org/0000-0002-3283-8807 


\section{Resumo}

Devido às mudanças do mercado e à necessidade do governo de fornecer educação aos cidadãos, o Brasil experimentou um processo de crescimento no ensino superior. Assim, de forma crescente, com transformações competitivas e constantes, as demandas por serviços de qualidade tornaram-se um denominador comum nas Instituições de Ensino Superior (IES), o qual expôs a necessidade de otimizar a gestão para que as instituições públicas melhorem seu desempenho em benefício dos estudantes. Ao contrário das outras empresas, que têm como objetivo a satisfação, essas IES geralmente percebem a satisfação como um meio para um fim. Ou seja, estudantes satisfeitos são estudantes leais. Assim, nesta pesquisa, o Modelo Europeu de Satisfação (ECSI) foi aplicado a 1.581 estudantes de 23 universidades públicas e livres no Brasil. Como resultado, observou-se que a satisfação é um fator determinante da lealdade. Entre os determinantes de satisfação, observou-se que a imagem, o tratamento das reivindicações e o valor percebido são os mais representativos. Dessa forma, a gestão eficiente da imagem da IES pública, o tratamento adequado das reivindicações dos estudantes e a percepção de que o investimento de esforço e tempo gerará ganhos futuros têm uma influência positiva e significativa na continuidade do aluno no mundo.

Palavras-chave: Ensino. Instituições Públicas de Ensino Superior. Alunos. Satisfação. Lealdade. ECSI.

\section{Abstract}

Due to market changes and government necessity to provide education to citizens, Brazil has been experiencing a process of growth in its higher education. Thus, in an increasingly competitive and transforming environment, the overall demand for qualified services have become a common denominator in Higher Education Institutions HEls. The later exposed the necessity for management optimization so that public institutions could improve their performance for the benefit of students. Unlike other companies that aim to satisfy themselves, these HEls often perceive satisfaction as a means to an end. That is, satisfied students are loyal students. Thus, in this research the European Satisfaction Model (ECSI) was applied to 1581 students from 23 public and free universities in Brazil. As a result, it has been observed that satisfaction is a determinant of loyalty. Among the determinants of satisfaction, it was observed that the image, the treatment of complaints and perceived value are the most representative key-factor elements. In this way, the efficient management of the public HEl image, the adequate treatment of students' complaints and the perception that the investment of effort and time will generate future gains has a positive and significant influence on the continuity of students in Brazilian HEls.

Keywords: Education. Public Higher Education Institutions. HEls. Students. Satisfaction. Loyalty. ECSI.

\section{INTRODUÇÃO}

Nas últimas décadas o setor de educação superior tem sofrido mudança ao redor do mundo (Alves \& Raposo, 2007). De forma análoga tem acontecido no Brasil, embora de forma mais recente (Santos, 2016). Por outro lado, a redução dos recursos públicos destinados a esse fim tem exigido maior responsabilidade por parte das instituições públicas em termos de qualidade e eficiência (Centre for Quality Assurance and Evaluation of Higher Education [CQAEHE] \& Comite National d'Evaluation [CNE], 1998). Por outra via, as instituições de 
ensino superior têm recebido, cada vez mais, diferentes tipos de estudantes, com um público cada vez mais diversificado, com ampla gama de necessidades, interesses e objetivos: jovens buscando sua formação inicial, adultos procurando atualização de seus conhecimentos, bem como pós-graduandos vislumbrando aprofundamento e especialização em determinadas áreas.

As instituições públicas são agora obrigadas a competir com maior número de instituições privadas, muito embora com recursos cada vez mais escassos. Ainda assim, no cenário atual brasileiro das IES públicas, a procura é muito maior que a oferta, reduzindo sensivelmente a necessidade de estratégias de marketing voltadas para a captação de alunos (Alves et al., 2010).

Porém, já se espera que no futuro haja um cenário cada vez mais competitivo, tendo em vista a política de investimento em bolsas de estudos e financiamento estudantil, ofertados por entidades governamentais, em detrimento do investimento nas universidades públicas, como forma de ampliação no número de vagas.

Nesse cenário, as universidades públicas menos competitivas perderiam parte de seus alunos, ou ainda deixariam espaço para as universidades particulares. Essa perda de espaço prejudicaria seu potencial de geração de pesquisas e criação de novas tecnologias, uma vez que, por exemplo, no Brasil, as Instituições de Ensino (IES) públicas esforçam-se para cumprir sua missão "ensino, pesquisa e extensão" e focam os seus esforços de comercialização para os resultados de suas pesquisas em "transferências de tecnologias" (Alves et al., 2010).

Segundo Ping (1993), uma forma de avaliar se uma instituição está sendo eficiente no cumprimento de suas funções é através da análise da satisfação e lealdade dos alunos. Nesse sentido, a avaliação do serviço que realizam e da maneira como é fornecido dá uma indicação da capacidade de como a instituição vai se desenvolver no futuro (Eskildsen et al., 1999).

Para Zink e Vob (1999), Rosa et al. (2000) e Alves e Raposo (2007), as instituições de ensino superior, inclusive as públicas, devem medir o nível de satisfação e lealdade de seus públicos-alvo, especialmente de seus alunos, sendo, portanto, um dos critérios essenciais para a excelência das instituições públicas (Alves \& Raposo, 2007). Apesar da sua importância, um conjunto limitado de pesquisas tem abordado a temática no contexto da educação superior pública e gratuita no Brasil (Santos, 2016). Dessa forma, tem-se o seguinte problema de pesquisa: quais são os determinantes da satisfação e lealdade discente em instituições públicas e gratuitas no Brasil?

\section{SATISFAÇÃO E LEALDADE NA EDUCAÇÃO}

Para competir de forma eficaz, uma instituição de ensino necessita diferenciar-se dos seus concorrentes (Joseph \& Joseph, 1997) por meio do desenvolvimento de uma imagem 
distintiva de valor, que seja comunicada com sucesso aos estudantes e empregadores através da satisfação e da lealdade (King, 1995). Assim, essa expressiva satisfação no ambiente educacional impulsionou a criação de diversos modelos de mensuração do nível de satisfação e lealdade (Alves \& Raposo, 2007), uma vez que mensurar a satisfação e a lealdade dos discentes se transformou em uma questão estratégica (Sun \& Kim, 2013).

Nesse sentido, Cheng e Tam (1997) definem, dentre as principais correntes teóricas de estudos de satisfação e lealdade, duas abordagens a serem avaliadas: o Modelo de Satisfação Americano e o Modelo de Satisfação Europeu, porém, em termos de avaliação da qualidade do ensino universitário, o modelo mais utilizado é o modelo Europeu (Brown \& Mazzarol, 2009; Chiandotto et al., 2007; Chitty \& Soutar, 2004; Eurico et al., 2018; Martensen et al., 2000; Turkyilmaz et al., 2018).

\subsection{MODELO DE SATISFAÇÃO EUROPEU}

O European Customer Satisfaction Index (ECSI) é um modelo de equações estruturais com variáveis latentes que vincula a satisfação dos estudantes aos seus fatores determinantes, e, por sua vez, a sua consequência: a lealdade (Martesen et al., 2000). Sua sistemática integra a satisfação do cliente como objetivo central na gestão das organizações, fornecendo um quadro de comunicação entre seus clientes, colaboradores e acionistas.

No modelo, o índice de satisfação do cliente é explicado por quatro construtos antecedentes: imagem, atendimento das expectativas, qualidade percebida e valor percebido, e por dois construtos consequentes: tratamento a reclamações e lealdade. Esses sete construtos do modelo estrutural são variáveis latentes e não podem servir como objeto de observação direta. Devem ser associados a um conjunto de indicadores "variáveis de medida", obtidos através da aplicação de questionário junto aos clientes (Lopes et al., 2009).

\subsection{A IMAGEM, A SATISFAÇÃO E A LEALDADE DISCENTE}

Para Wilkie (1994), o comportamento do usuário é uma atividade emocional que objetiva satisfazer uma necessidade ou um desejo. Já autores como Boulding (1968), Kontz et al. (2014) e Moscovici (1978) afirmam que a sua escolha é influenciada pelas imagens que a pessoa possui perante o serviço/produto oferecido e o benefício que lhe será proporcionado.

Assim, perante as mudanças que ocorrem na sociedade, faz-se necessária uma atenção voltada ao posicionamento e à comunicação com o público-alvo. Para tanto, independentemente do ramo, atividade ou porte, as organizações, para sobreviver, necessitam estar atentas à sua imagem. Mais precisamente, as Instituições de Ensino Superior devem 
adaptar suas estruturas a essas novas exigências para que possam sobreviver num ambiente cada vez mais competitivo (Neves \& Ramos, 2002).

Para Grönroos (1997), uma imagem favorável e bem conhecida, corporativa e/ou local, é um patrimônio para qualquer instituição, porque a imagem tem um impacto sobre a visão que o público-alvo tem da comunicação e das operações da instituição. Assim, para ser bemsucedida, uma instituição educacional deve lidar eficazmente com seus públicos e gerar alto nível de satisfação e lealdade. Dessa forma, torna-se importante entender o comportamento dos alunos por meio da imagem que estes fazem da IES, o que permite identificar a maneira como decidem entre as alternativas de serviços e como avaliam esses serviços (satisfação), uma vez que os tenham recebido (Tomio \& Souza, 2007).

Palacio et al. (2002) corroboram essa ideia dizendo que o estabelecimento de uma imagem positiva para uma universidade, através de uma variedade de meios, é essencial para o recrutamento de novos alunos. Além disso, uma boa imagem da instituição reflete em alunos satisfeitos e leais, com maiores habilidades e conhecimento (Justin et al., 2019; Letcher \& Neves, 2010; Masserini et al., 2018).

Portanto, sendo a imagem uma variável que visa integrar todo o tipo de associações que os clientes fazem de uma empresa/marca, utilizando a informação proveniente de várias fontes ao longo do tempo (Biégas, 2009), as instituições de ensino superior devem assumir a iniciativa de criar uma boa imagem, visto que ela possui um impacto significativo na formação da satisfação e da lealdade (Alves \& Raposo, 2007).

\subsection{EXPECTATIVAS E A SATISFAÇÃO DISCENTE}

As expectativas com relação à qualidade dos produtos ou serviços constituem o terceiro determinante ou antecedente da satisfação. Elas referem-se ao conhecimento acumulado sobre a qualidade das ofertas de um fornecedor. Assim como a qualidade atual tende a influenciar a satisfação do usuário, as experiências passadas também são responsáveis por essa influência, e ambas são representadas pelas expectativas (Anderson et al., 1994).

Segundo Fornell et al. (1996), as expectativas representam as experiências de consumo anteriores - incluindo as adquiridas através de propaganda boca a boca - e a previsão da capacidade de o fornecedor proporcionar produtos de qualidade no futuro. Churchill e Surprenant (1982) afirmam que as expectativas refletem o desempenho antecipado do produto ou serviço.

São várias as expectativas que os públicos-alvo de uma instituição podem ter sobre um produto ou serviço, e estas variam e dependem do foco dado às necessidades dos diferentes 
grupos envolvidos (Kotler, 1998). No entanto, no âmbito acadêmico, as expectativas dos alunos com relação à educação superior são altas (Davies, 2002).

A fim de ser bem-sucedida, uma instituição educacional necessita lidar eficazmente com seus públicos a fim de gerar um alto nível de satisfação (Neves \& Ramos, 2002). Palacio et al. (2002) mostraram que a satisfação é uma resposta afetiva em determinado tempo e é determinada pelas expectativas dos estudantes quando entram na universidade.

Para Neves e Ramos (2002), as instituições privadas que há alguns anos atuavam de forma passiva nas questões educacionais, principalmente nas relações com o mercado, hoje são forçadas, dado o aumento da competitividade, a ser proativas em suas ações estratégicas, especialmente na identificação e satisfação das expectativas. Porém, a educação superior pública brasileira adota uma abordagem de dentro para fora, presumindo que os gestores sabem o que os alunos esperam e querem da instituição. Mas as indústrias bem-sucedidas têm utilizado a abordagem de fora para dentro, visando ao atendimento das expectativas dos seus públicos-alvo com base em pesquisas sobre o que eles esperam do seu serviço (Sander et al., 2000).

\subsection{A QUALIDADE E A SATISFAÇÃO PERCEBIDA}

Para Kotler (1994), satisfação é o nível de sentimento de uma pessoa resultante da comparação do desempenho (ou resultado) de um produto em relação a suas expectativas. Mowen (1995) aponta a satisfação como a atitude geral referente a um produto ou serviço depois de sua aquisição e uso.

Já a qualidade percebida é definida como "o julgamento sobre a superioridade ou excelência global de um serviço" (Zeithaml, 1987) e pode ser, muitas vezes, confundida com satisfação, pois as duas são intangíveis (Kotler, 1998). A primeira é percebida como uma forma de atitude (Olshavsky \& Rosen, 1985), enquanto a segunda é vista como resultado de uma experiência de consumo (Marchetti \& Prado, 2004).

A qualidade percebida é um conceito complexo e tem sido definida como uma relação entre as expectativas e a performance percebida de um serviço (Grönroos, 1997). Parasuraman e Grewal (2000) a definem como um juízo global, ou atitude, relativo à superioridade de um serviço ou produto. Ou seja, essa qualidade é o que o usuário espera que seja fornecido pela empresa, ao passo que a satisfação é o resultado da comparação entre o desempenho esperado e o desempenho real. Assim, as expectativas assumem relevância como determinante da satisfação e estão positivamente associadas à qualidade percebida (Gonçalves et al., 2010). 


\subsection{VALOR PERCEBIDO E SATISFAÇÃO}

Zeithaml (1987) define o valor percebido como uma avaliação geral realizada pelo usuário perante a utilidade de um produto baseado em percepções do que é recebido e do que é dado. Sheth et al. (1991) retificam que o valor percebido por ele é uma análise dos benefícios agregados pelos serviços ou produtos adquiridos em relação às necessidades e desejos. Já Choi et al. (2004) o define como uma construção cognitiva que necessita de um trade-off entre benefícios e sacrifícios.

Ainda, Leblanc e Nguyen (1999) e Costa (2007) desenvolveram análises direcionadas à perspectiva dos estudantes associada ao serviço da instituição de ensino e tiveram o valor como multidimensional. Segundo Sheth et al. (1991), há circunstâncias em que uma determinada escolha de consumo está condicionada a algo em especial, sem o qual tal decisão seria relativizada.

Ou seja, o valor percebido é dado como um valor condicional como resultado de uma situação específica. Nesse sentido, Petrick (2002, 2004) descreve que, no contexto educacional, a fim de representar o valor percebido, têm-se como sendo o preço e outros fatores não monetários o tempo e o esforço.

\subsection{LEALDADE}

A lealdade pode ser definida como a intenção de realizar um conjunto variado de comportamentos que manifestam a motivação para a relação de troca, como a compra repetida e o boca a boca positivo (Lam et al., 2004). Diversas pesquisas demonstram que os usuários percebem dois meios para a geração da lealdade: o encantamento (Lee et al., 2001) e a percepção de alto valor proveniente de excelentes serviços e produtos de qualidade (Parasuraman \& Grewal, 2000).

Em seu estudo, Agustin e Singh (2005) descobriram que a lealdade do estudante vem se tornando interesse direto e um dos objetivos principais das instituições universitárias, pois usuários leais são mais propensos a buscar benefícios em longo prazo e envolver-se em ações de cooperação que favoreçam ambos os lados.

Por outro lado, tem-se que a evasão escolar é uma consequência direta do baixo nível de satisfação e de lealdade discente. Assim, pelo princípio constitucional da eficiência na administração pública, na busca pela melhor utilização possível dos recursos públicos, as instituições de ensino públicas deveriam buscar entender e gerenciar seus determinantes de maneira a se evitarem desperdícios e garantir maior rentabilidade social. 


\subsection{RECLAMAÇÕES}

As reclamações são um conflito estabelecido entre os usuários e as instituições de ensino, sendo uma expressão de insatisfação decorrente de um problema com um produto ou serviço (Landon, 1980). Também podem ser consideradas como o resultado de uma avaliação negativa entre as percepções e as expectativas dos consumidores, ou seja, o boca a boca, conhecido como word of mouth (WOM), é avaliado como negativo (Anderson et al., 1994).

Existe um forte conhecimento sobre fatores que podem levar a reclamações dos usuários. Um importante fator que gera essas reclamações é o desempenho não alcançado dos produtos ou serviços e, consequentemente, a insatisfação do usuário. Usuários insatisfeitos são mais propensos a se queixar do que consumidores satisfeitos (Fornell et al., 1996).

No entanto, esse boca a boca pode ser considerado positivo quando representado por recomendações, as quais são feitas geralmente através de clientes satisfeitos. Pate (1990) diz que, durante a sua carreira universitária, a satisfação dos alunos em suas universidades tem efeito positivo sobre a sua vontade de recomendar a escola após a graduação. Sob o mesmo ponto de vista, recomendações positivas de atuais estudantes são, muitas vezes, suficientes para cultivar o interesse de novos alunos para uma determinada instituição (Palacio et al., 2002).

Dessa forma, alguns autores, como Alves e Raposo (2007), utilizam o WOM, em vez de reclamações, como construto na avaliação da satisfação dos clientes. Independentemente, esse construto é fundamental para alcançar níveis crescentes de satisfação dos alunos, gerando uma expressiva vantagem competitiva, tanto com relação ao desempenho dos atuais acadêmicos quanto ao marketing boca a boca positivo gerado, que é indispensável para a conquista de novos alunos (Wilkins \& Balakrishnan, 2013).

\subsection{SATISFAÇÃO DISCENTE COMO FERRAMENTA DE DESENVOLVIMENTO REGIONAL}

A universidade contribui com a transformação social e econômica da região em que está localizada a partir da sua busca para as questões locais, tanto por meio do desenvolvimento de conhecimentos científicos e tecnológicos quanto da capacitação dos recursos humanos. Ełzkowitz (2009) sugere que o desenvolvimento econômico de um país ou região está vinculado às suas instituições de ensino e seu papel de articulação com as empresas locais.

A universidade cria um ambiente de desenvolvimento regional e local a partir da articulação de todos os atores envolvidos no processo do seu funcionamento (Goebel \& Miúra, 2004). Os montantes financeiros alocados para o pagamento de seus funcionários, a 
manutenção da estrutura física e a contratação de prestadores de serviços geram grandes somas de dinheiro injetado na economia, o que, para Kontz et al. (2014), "trata-se de um efeito multiplicador por intermédio do qual o dinheiro despendido em uma atividade é transferido para outras atividades, sobretudo aquelas que lhe estão relacionadas."

Nessa lógica de desenvolvimento regional a partir do papel das universidades a satisfação dos alunos passa a ter um importante papel. Existe uma relação direta entre a satisfação do aluno e a sua permanência no ensino, e quanto mais alunos satisfeitos, maior é o índice de sucesso do curso, atraindo mais alunos para a universidade e criando um ciclo de desenvolvimento local e regional.

\section{METODOLOGIA}

Nesta pesquisa utilizou-se uma abordagem objetivista, de cunho quantitativo. Quanto aos objetivos, a pesquisa foi tratada como descritiva. Quanto aos procedimentos e técnicas utilizadas, a pesquisa foi classificada como de levantamento com amostragem por conveniência. $O$ instrumento de coleta de dados utilizado foi um questionário autoadministrado adaptado de Alves e Raposo (2007), em que se utilizou uma escala Likert que variou entre um (discordo totalmente) e dez (concordo totalmente). $O$ modelo testado nesta pesquisa é demonstrado na Figura 1.

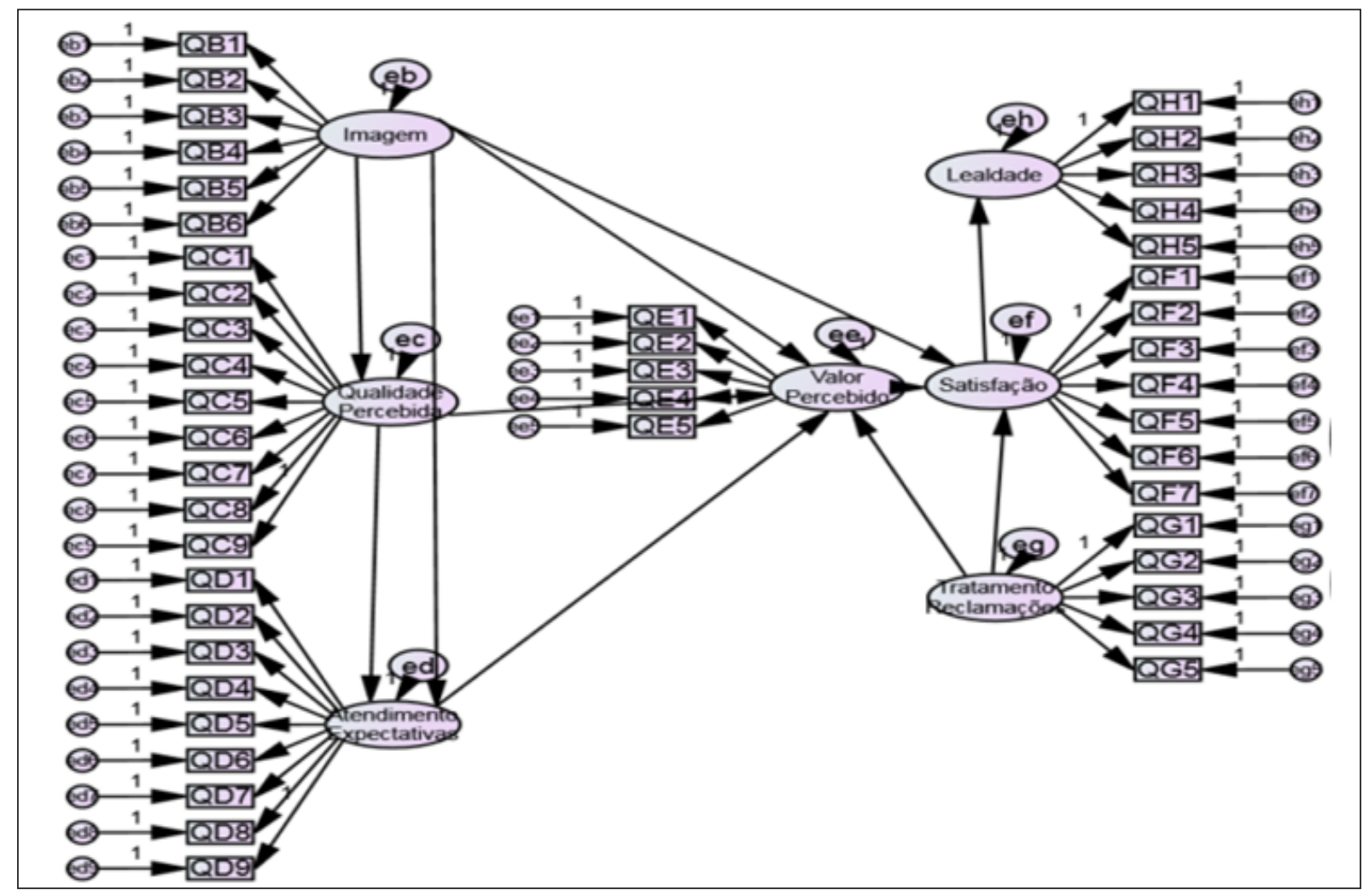

Figura 1. Modelo ECSI proposto 
Observa-se na Figura 1 que a satisfação e a lealdade dos alunos estão relacionadas às variáveis antecedentes - imagem, expectativa dos alunos, qualidade percebida e valor percebido - e às variáveis consequentes - lealdade e comunicação boca a boca.

Primeiramente, a fim de alcançar resultados mais confiáveis, foi realizado o tratamento preliminar dos dados antes da aplicação das técnicas estatísticas multivariadas, visto que Hair et al. (2010) recomendam que a ocorrência de valores omissos seja verificada quanto à magnitude (extensão de ocorrência) e à aleatoriedade de distribuição.

Além disso, Malhotra (2011) recomenda que os questionários com 10\% das questões não respondidas sejam eliminados. E que aqueles que apresentarem menos de $10 \%$ de valores omissos sejam mantidos na pesquisa. Por fim, o tratamento dos dados buscará identificar a existência de outliers multivariados através do teste de Hadi.

A fim de validar cada um dos construtos estudados e também o modelo proposto, foi aplicada a técnica de modelagem de equações estruturais. Esta é uma metodologia multivariada que, ao combinar aspectos da regressão múltipla com análise fatorial, possibilita estimar, de forma simultânea, uma série de relações de dependência (Hair et al., 2010).

Para tanto, primeiramente, verificou-se a existência de estimativas transgressoras como, por exemplo, a presença de variâncias negativas, correlações entre construtos muito próximos a 1 e erros padrão muito elevados (Cavalheiro, 2015). Caso se tenha a confirmação da inexistência de estimativas transgressoras, a qualidade de ajuste geral do modelo deverá ser avaliada, pois, de acordo com Schumacker e Lomax (2004), ela determina o quanto o modelo de equações estruturais se ajusta aos dados da amostra.

No processo de validação do modelo estrutural considerou-se um modelo bem ajustado em que o qui-quadrado fosse superior a $5 \%$ ou, então, que a razão qui-quadrado/ graus de liberdade fosse menor que cinco (Hopper et al., 2008). Para os índices CFI, GFI, NFI e TLI, utilizaram-se valores maiores que 0,95 (Byrne, 2013; Hooper et al.), o RMSEA deveria ser inferior a 0,08 (Thompson, 2004), e o RMSEA deveria ser menor que 0,05 (Al-Refaie et al., 2011; Hooper et al.).

\section{RESULTADOS}

Obtiveram-se 1.581 questionários válidos de 23 universidades públicas e gratuitas brasileiras, sendo elas: FURG (19) alunos, IFSul (2), UEL (1), UERJ (36), UFBA (10), UFF (16), UFJF (4), UFMG (32), UFOP (11), UFPB (11), UFPE (17), UFPel (894), UFPR (3), UFRGS (184), UFSC (9), UFSJ (3), UFSM (33), UFV (57), UNB (11), UNICAMP (3), UFCG (2), UFV (10) e Udesc (213). Na Figura 2 apresenta-se o resultado da pesquisa. 


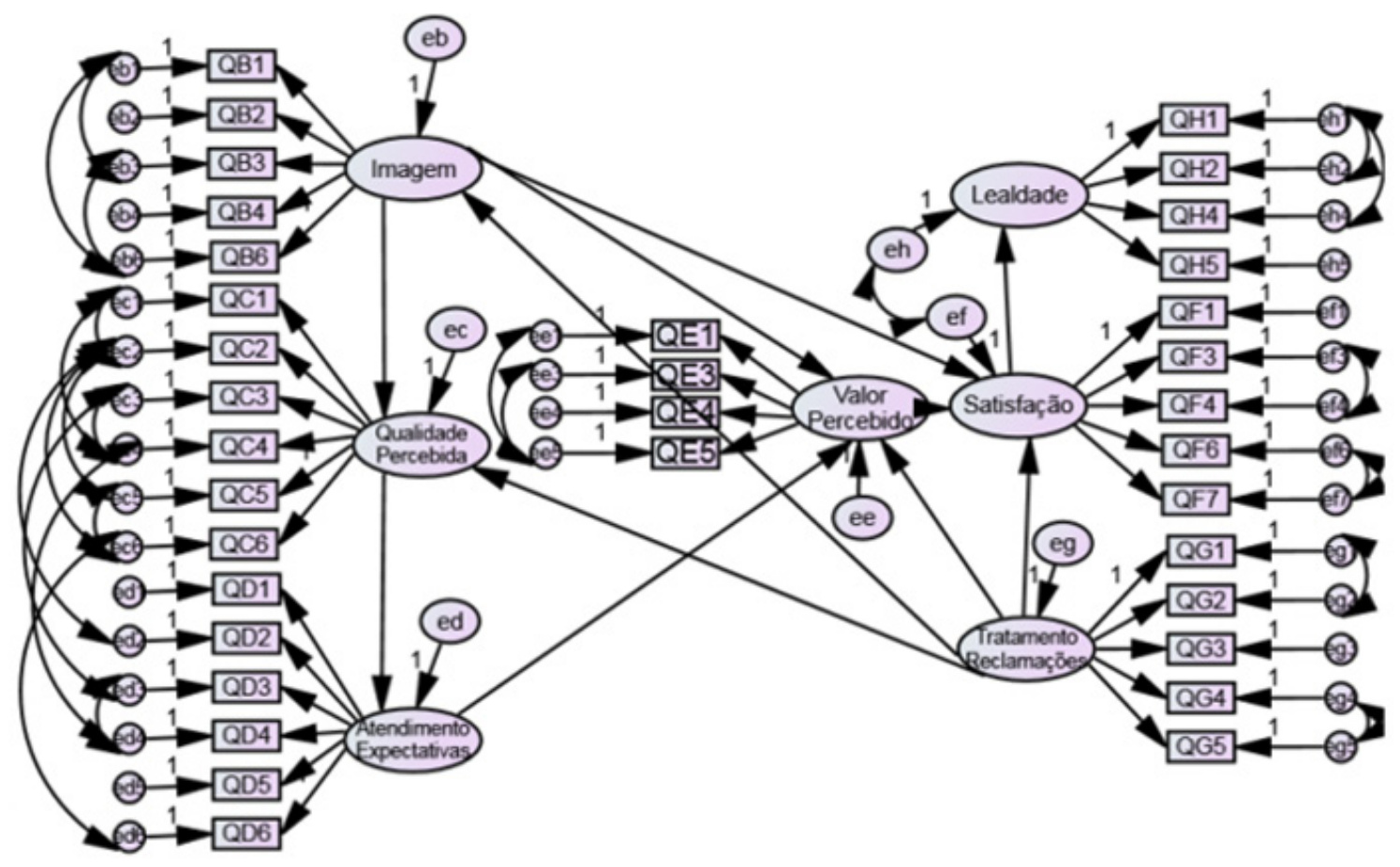

Figura 2. Modelo ECSI final

Observa-se à esquerda da Figura 2 o modelo de avaliação da lealdade e satisfação discente (ECSI) proposto por Alves e Raposo (2007). Como o modelo não se mostrou válido, procedeu-se pela estratégia de exclusão das variáveis não significativas ou pouco contributivas e de inclusão de covariâncias entre os erros das variáveis e dos construtos. $\mathrm{Na}$ direita da Figura tem-se o modelo final da pesquisa. Na Tabela 1 apresentam-se os índices de ajuste do modelo estrutural.

Tabela 1

Índices de ajustamento do modelo integrado - modelo inicial e modelo final

\begin{tabular}{lrr}
\hline \multicolumn{1}{c}{ Índices de ajuste } & \multicolumn{1}{c}{ Valores Iniciais } & \multicolumn{1}{c}{ Valores Finais } \\
\hline Qui-quadrado (valor) & 8030,425 & $1.944,684$ \\
Qui-quadrado (probabilidade) & 0,000 & 0,000 \\
Graus de Liberdade & 978,000 & 526,000 \\
Qui-quadrado / Graus de Liberdade & 8,211 & 3,697 \\
GFI - Goodness of Fit & 0,842 & 0,952 \\
CFI - Comparative Fit Index & 0,896 & 0,971 \\
NFI - Normed Fit Index & 0,961 \\
TLI - Tucker-Lewis Index & 0,883 & 0,967 \\
RMSEA - Root Mean Square Error of Approximation & 0,890 & 0,034 \\
RMR - Root Mean Square Residual & 0,055 & 0,080 \\
\hline
\end{tabular}


Mediante os dados obtidos é possível observar, na Tabela l, que todos os índices (razão Qui-quadrado / Graus de Liberdade, CFI, GFI, NFI, TLI, RMSEA, RMR) foram significativos após os ajustes realizados.

Na Tabela 2 apresentam-se os pesos padronizados das regressões.

Tabela 2

Pesos padronizados das regressões

\begin{tabular}{|c|c|c|c|c|c|c|c|}
\hline Construto & Dir. & Construto & Peso & Construto & Dir. & Construto & Peso \\
\hline $\begin{array}{l}\text { Qualidade Perce- } \\
\text { bida }\end{array}$ & $<--$ & Imagem & 0,71 & Valor Percebido & $<--$ & $\begin{array}{l}\text { Tratamento às } \\
\text { Reclamações }\end{array}$ & 0,13 \\
\hline $\begin{array}{l}\text { Qualidade Perce- } \\
\text { bida }\end{array}$ & $<--$ & $\begin{array}{l}\text { Tratamento às Re- } \\
\text { clamações }\end{array}$ & 0,26 & Satisfação & $<--$ & $\begin{array}{l}\text { Tratamento às } \\
\text { Reclamações }\end{array}$ & 0,17 \\
\hline $\begin{array}{l}\text { Atendimento às } \\
\text { Expectativas }\end{array}$ & $<--$ & $\begin{array}{l}\text { Qualidade Perce- } \\
\text { bida }\end{array}$ & 0,93 & Satisfação & $<--$ & Valor Percebido & 0,52 \\
\hline Valor Percebido & $<--$ & $\begin{array}{l}\text { Atendimento às } \\
\text { Expectativas }\end{array}$ & 0,29 & Satisfação & $<--$ & Imagem & 0,34 \\
\hline Valor Percebido & $<--$ & Imagem & 0,51 & Lealdade & $<--$ & Satisfação & 0,90 \\
\hline Imagem & $<--$ & $\begin{array}{l}\text { Tratamento às Re- } \\
\text { clamações }\end{array}$ & 0,61 & & & & \\
\hline
\end{tabular}

Observa-se, na Tabela 2, que o único determinante da Lealdade Discente nas organizações públicas investigadas foi o construto Satisfação, em que uma unidade da satisfação discente aumenta 0,90 unidade da lealdade discente. Tal resultado denota a alta importância do acompanhamento do nível de satisfação dos alunos como forma de instigá-los a terminar seu curso e melhorar os índices de permanência e êxito. Assim, para ser bem-sucedida e eficiente na sua atividade-fim, uma instituição educacional, embora pública e gratuita, deve lidar eficazmente com seus públicos e gerar alto nível de satisfação e, por consequência direta, a lealdade discente.

Por outro lado, observa-se, na Tabela 2, que os maiores determinantes da satisfação discente foram Imagem, Tratamento às Reclamações e Valor Percebido, com carga total padronizada (direta e indireta) igual a 0,70, 0,70 e 0,52 para aqueles construtos, respectivamente. Dessa forma, torna-se importante compreender o comportamento dos alunos por meio da imagem que estes fazem da IES, o que permite identificar a maneira como decidem entre as alternativas de serviços e como avaliam esses serviços, uma vez que os tenham recebido.

Diversas evidências confirmam essa ideia, apontando que o estabelecimento de uma imagem positiva para uma universidade é essencial para o recrutamento de novos alunos (Palacio et al., 2002). Além disso, uma boa imagem da instituição poderia refletir em alunos satisfeitos e leais, com maiores habilidades e conhecimentos (Letcher \& Neves, 2010). 
Ademais, mostrou-se relevante na pesquisa o tratamento às reclamações. Um importante fator que gera essas reclamações é o desempenho não alcançado dos produtos ou serviços, neste caso, o processo de ensino-aprendizagem e todos os requisitos de qualidade de ensino e de relacionamento professor-aluno que advêm dele. Observa-se no modelo final que o tratamento adequado às reclamações reforça positivamente a imagem da instituição e do curso, bem como o valor percebido e o nível da satisfação do aluno. Por outra via, o tratamento inadequado reforça negativamente tais construtos, reduzindo o nível de lealdade discente. Torna-se, portanto, estratégico à gestão pública o tratamento adequado das reclamações, bem como a antecipação de tais problemas como forma de evitar tais reclamações.

Por fim, o construto Valor Percebido também foi relevante nesta pesquisa. De forma geral, a literatura conceitua o valor percebido como sendo uma avaliação geral realizada pelo usuário perante a utilidade de um produto baseado em percepções do que é recebido e do que é dado, como sendo uma consequência da análise dos benefícios agregados pelos serviços ou produtos adquiridos com relação às necessidades e aos desejos dos indivíduos (Sheth et al., 1991; Zeithaml, 1987).

Trata-se, portanto, de uma construção cognitiva que necessita de um trade-off entre benefícios e sacrifícios (Choi et al., 2004). Nesse sentido, Petrick $(2002,2004)$ descreve que, no contexto educacional público e gratuito, a fim de representar o valor percebido, têm-se como fatores não monetários o tempo, o esforço e as expectativas de benefícios futuros que advêm deles. Nesse sentido, torna-se importante à gestão das universidades públicas brasileiras o acompanhamento dessa medida, assim como ações positivas as quais podem reforçar, junto aos discentes, o potencial de geração de valor futuro aos alunos no que tange à conclusão de seus estudos ou de sua especialização em determinada área.

\section{CONSIDERAÇÕES FINAIS}

As instituições de ensino superior têm recebido, cada vez mais, diferentes tipos de estudantes, com um público cada vez mais diversificado, com ampla gama de necessidades, interesses e objetivos: jovens buscando sua formação inicial, adultos procurando atualização de seus conhecimentos, bem como pós-graduandos vislumbrando aprofundamento e especialização em determinadas áreas.

Vive-se em um contexto competitivo em que se torna importante entender o comportamento dos alunos a fim de identificar o nível de satisfação e lealdade desses discentes perante suas IES (Tomio \& Souza, 2007). A satisfação é um fator fundamental para gerar e manter a lealdade do aluno ao longo da sua formação, influenciando fortemente no seu aprendizado. Assim, o desenvolvimento de pesquisas orientadas a uma melhor 
identificação das percepções dos alunos e de suas necessidades pode proporcionar às IES melhores condições de decisão quanto às suas ações de desenvolvimento (Bronnemann, 2002). Como consequência, pesquisas relacionadas à avaliação da qualidade de serviços nas IES têm recebido a atenção de muitos pesquisadores (Santos, 2016).

Em meio a essa demanda, após a constatação, por meio da investigação teórica e empírica, de que o modelo europeu de satisfação seria o modelo mais adequado ao escopo do presente estudo, a partir da escala ECSI adaptada de Alves e Raposo (2007), procurouse validar um modelo que poderia explicar a satisfação e a lealdade discentes perante as instituições de ensino superior públicas e gratuitas no Brasil, visto que as universidades visam à obtenção de um corpo discente leal.

A pesquisa foi de cunho quantitativo, baseada na indução, buscando a generalização mediante a coleta, o exame e a análise de dados específicos. Sendo do tipo survey, ela contou com a participação de 1.796 respondentes, com 1.574 questionários válidos. Com a validação do modelo integrado e a partir da verificação dos pesos obtidos na integração de cada construto, chegou-se ao resultado final do estudo.

Como resultado, observou-se que o principal determinante da lealdade dos discentes é a sua satisfação para com a IES. Essa satisfação ocorre na medida em que os discentes têm suas necessidades atendidas, gerando, assim, uma boa imagem da IES. O que leva à afirmativa de que a satisfação ocorre por meio da imagem que os alunos possuem de sua instituição. Atrelada ao construto imagem, te-se como fator significativo a inovação. Esta deve ser valorizada e incentivada pela universidade para que a melhoria contínua possa se instalar como prática corrente na instituição, gerando melhor preparação dos discentes (Morozini \& Audy, 2010). Além disso, como se pode- observar no modelo validado, no que se refere à explicação do nível de satisfação e lealdade discente, têm-se os construtos valor percebido e o correto tratamento das reclamações.

Para o valor percebido, tem-se como fator significativo a possibilidade de a IES inserir o discente no mercado de trabalho. Uma vez que, considerando que o objetivo final de uma instituição de ensino é ter o aluno formado, capacitado e habilitado a exercer a profissão para a qual se preparou, nada mais relevante, portanto, do que analisar a opinião dos empregadores dessa mão de obra formada (Helena, 2001).

Já para o tratamento das reclamações, vê-se o rápido atendimento das reclamações dos usuários gerando efeito positivo na recomendação de sua IES. Pate (1990) diz que durante a sua carreira universitária, a satisfação dos alunos em suas universidades tem efeito positivo sobre a sua vontade de recomendar a escola após a graduação. Sob o mesmo ponto de vista, recomendações positivas de atuais estudantes são, muitas vezes, suficientes para cultivar o interesse de novos alunos para uma determinada instituição (Palacio et al., 2002). 
Conclui-se, assim, que o modelo forneceu uma eficaz estrutura para examinar as maneiras como a lealdade do estudante é gerada, a qual está diretamente ligada á satisfação, como resposta ao problema de pesquisa; além disso, os fatores determinantes dessa satisfação estão relacionados à imagem, seguida do valor percebido e do atendimento das reclamações. Nesse sentido, a presente pesquisa sugere que as instituições de ensino públicas deveriam buscar entender e gerenciar os determinantes da Lealdade e da Satisfação. Sugere-se, ainda, para pesquisas futuras a ampliação da amostra, bem como a comparação dos escores entre universidades públicas e gratuitas e universidades particulares no Brasil.

\section{REFERÊNCIAS}

Agustin, C., \& Singh, J. (2005). Curvilinear effects of consumer loyalty determinants in relational exchanges. Journal of Marketing Research, 42(1), 96-108.

Alves, H., \& Raposo, M. (2007). Student satisfaction index in Portuguese public higher education. The Service Industries Journal, 27(6), 795-808.

Alves, H., Raposo, M., \& Mainardes, E. (2010). O marketing no ensino superior: Comparativo Brasil-Portugal. Revista de Administração FACES Journal, 9(4).

Al-Refaie, A., Ghnaimat, O., \& Ko, J.-H. (2011). The effects of quality management practices on customer satisfaction and innovation: A perspective from Jordan. International Journal of Productivity and Quality Management, 8(4), 398-415.

Anderson, E. W., Fornell, C., \& Lehman, D. R. (1994). Customer satisfaction, market share, and profitability. Journal of Marketing, 58(1), 53-66.

Biégas, S. (2009). A qualidade do relacionamento e a lealdade no comércio eletrónico. (Tese de Mestrado, Universidade Federal do Paraná, Centro de Pesquisa e Pós-graduação em Administração, Curitiba, Paraná, Brasil).

Boulding, K. E. (1968). The image. Ann Arbor Paperback..

Bronnemann, M. R. (2002). Marketing em instituições de ensino superior: A promoção do processo seletivo. (Dissertação de Mestrado, Universidade Regional de Blumenau, Blumenau, Santa Catarina, Brasil).

Brown, R. M., \& Mazzarol, T. W. (2009). The importance of institutional image to student satisfaction and loyalty within higher education. Higher Education, 58, 81-95. 
Byrne, B. M. (2013). Structural equation modeling with AMOS: Basic concepts, applications, and programming. Routledge.

Cavalheiro, E. (2015). Determinantes da estrutura de capital da micro e pequenas empresas: Uma abordagem comportamental. (Tese de Doutorado, Universidade Federal de Santa Maria, Santa Maria, Rio Grande do Sul, Brasil).

Cheng, Y. C., \& Tam,W. M. (1997). Multi-models of quality in education. Quality Assurance in Education, 5(1).

Chiandotto, B., Bini, M., \& Bertanccini, B. (2007). Quality assessment of the university educational process: An application of the ECSI model. In L. Fabbris, Effectiveness of university education in Italy (pp. 43-54). Heidelberg.

Chitty, B., \& Soutar, G. N. (2004). Is the European customer satisfaction index model applicable to tertiary education. Proceedings do ANZMAC 2004 Conference Wellington, Australian and New Zealand Marketing Academy, Wellington, 1-7.

Choi, K. S., Cho, W.-H., Lee, S., Lee, H., \& Kim, C. (2004). The relationships among quality, value, satisfaction and behavioral intention in health care provider choice: A South Korean study. Journal of Business Research, 57(8), 913-921.

Churchill, G. A., Jr., \& Surprenant, C. (1982). An investigation into the determinants of customer satisfaction. Journal of Marketing Research, 19, 491-504.

Costa, F. J. (2007). A influência do valor percebido pelo cliente sobre os comportamentos de reclamação e boca a boca: Uma investigação em cursos de pós-graduação lato sensu. (Tese de Doutorado, Fundação Getúlio Vargas, Escola de Administração de Empresas de São Paulo, São Paulo).

Centre for Quality Assurance and Evaluation of Higher Education, \& Comite National d'Evaluation. (1998, September). Evaluation of European education: A status report. Report prepared for the European Commission, DG XXII.

Davies, S. (2002). Marketing in higher education: Matching promises and reality to expectations. Responding to Student Expectations, OECD report, pp. 103-114.

Eskildsen, J., Martensen, A., Gronholdt, L., \& Kristensen, K. (1999, August 30-31). Benchmarking student satisfaction in higher education based on the ECSI methodology. Proceedings of the TQM for Higher Education Institutions Conference: Higher Education Institutions and the Issue of Total Quality, Verona, 385-402. 
Eskildsen, J., Martensen, A., Gronholdt, L. and Kristensen, K. (1999) Benchmarking student satisfaction in higher education based on the ECSI methodology. In Proceedings of the TQM for Higher Education Institutions Conference: Higher Education Institutions and the Issue of Total Quality, Verona, 30 - 31 August, pp.385-402.

Eurico, S., Pinto, P., Silva, J. A., \& Marques, C. (2018). The ECSI model in higher education in tourism: A segmentation analysis in the Portuguese case. Turizam: Medunarodni znanstveno-stručni časopis, 66(2), 208-226.

Etzkowitz, H. (2009). Hélice tríplice: Universidade-indústria-governo: Inovação em movimento. EDIPUCRS.

Fornell, C., Johnson, M. D., Anderson, E. W., Cha, J., \& Bryant, B. E. (1996) The American customer satisfaction index: Nature, purpose, and findings. Journal of Marketing, 60(4), 7-12.

Goebel, M. A., \& Miúra, M. N. (2004). A universidade como fator de desenvolvimento: Caso do município de Toledo-PR. Revista Expectativa, 3(3).

Gonçalves, E., Souza, J. C., Anunciação, L. P., Cruz, M. C. H., \& Teixeira, E. S. (2010). Gestão da qualidade do atendimento ao cliente: Aplicação do método SERVIQUAL para mensuração da qualidade dos serviços prestados pela Fisiomed. Revista Eletrônica Gestão e Negócios, 1(1).

Grönroos, C. (1997). Keynote paper from marketing mix to relationship marketing: Towards a paradigm shift in marketing. Management Decision, 35(4), 322-339.

Hair, J. F., Anderson, R., Tatham, R., \& Black, W. (2010). Análise multivariada de dados (6a ed.). Bookmam.

Helena, L. A. Satisfação do aluno: Satisfação do aluno, enquanto cliente, nas Instituições de Ensino Superior (IES). Anais do Congresso ENANGRAD, São Paulo, Brasil, 12.

Hopper, D., Coughlan, J., \& Mullen, M. (2008). Structural equation modelling: Guidelines for determining model fit. Electronic Journal of Businness Research Methods, 6(1).

Joseph, M., \& Joseph, B. (1997). Service quality in education: A student perspective. Quality Assurance in Education, 5(1), 15-21.

Justin, L. D., Jin, S., \& Hubert, V. (2019). Effect of international students' perceived service quality on the student's motivation, satisfaction, loyalty, and institutional image in higher education in China. International Journal of Science and Business, 3(2), 110-125. 
King, R. (1995). What is higher education for? Strategic dilemmas for the twenty-first century university. Quality Assurance in Education, 3(4), 14-20.

Kontz, L., Schuler, I., \& Palmeira, E. (2014). Turismo estratégico: Estudo sobre o potencial de desenvolvimento turístico a partir da criação de um parque temático. Revista Turismo y Desarrollo- TURYDES.

Kotler, P. (1994). Marketing Management: Analysis, planning, implementation and control. Prentice Hall.

Kotler, P. (1998). Administração de marketing: Análise, planejamento, implementação e controle ( 5 a ed.). Atlas.

Lam, S. Y., Shankar, V., Erramilli, M. K., \& Murthy, B. (2004). Customer value, satisfaction, loyalty, and switching costs: An illustration from a business-to-business service context. Journal of the Academy of Marketing Science, 32(3), 293-311.

Landon, E. L., Jr. (1980). The direction of consumer complaint research. Advances in Consumer Research, 7(1).

Leblanc, G., \& Nguyen, N. (1999). Listening to the customer's voice: Examining perceived service value among business college students. The International Journal of Educational Management, 13(4), 187-198.

Lee, J., Lee, J., \& Feick, L. (2001). The impact of switching costs on the customer satisfaction-loyalty link: Mobile phone service in France. Journal of services marketing, 15(1), 3548.

Letcher, D. W., \& Neves, J. S. (2010). Determinants of undergraduate business student satisfaction. Research in Higher Education Journal, 6(1), 1-26.

Lopes, H. E. G., Pereira, C. C. P., \& Vieira, A. F. S. (2009). Comparação entre os modelos norteamericano (ACSI) e Europeu (ECSI) de satisfação do cliente: Um estudo no setor de serviços. Revista de Administração Mackenzie, 10(1).

Malhotra, N. K. (2011). Pesquisa de marketing: Foco na decisão (3a ed.). Pearson Prentice Hall.

Marchetti, R., \& Prado, P. (2004, outubro-dezembro). Avaliação da satisfação do consumidor utilizando o método de equações estruturais: um Modelo aplicado ao setor elétrico brasileiro. RAC, 8(4), 9-32. 
Martensen, A., Gronholdt, L., Eskildsen, J. K., \& Kristensen, K. (2000). Measuring student oriented quality in higher education: Application of the ECSI methodology. Sinergie Rapporti di Ricerca, 9, 371-383.

Masserini, L., Bini, M., \& Pratesi, M. (2018). Do quality of services and institutional image impact students' satisfaction and loyalty in higher education?. Social Indicators Research, 1-25.

Moscovici. S. (1978). A representação social da psicanálise. Zahar.

Mowen, J. C. (1995). Consumer behavior (4a ed.). Englewood Cliffs: Prentice-Hall.

Neves, A. B., \& Ramos C. F. (2002). A imagem das instituições de ensino superior e a qualidade do ensino de graduação: $A$ percepção dos acadêmicos do curso de administração. Revista de Economia e Administração, 1(1), 75-84.

Olshavsky, R. W., \& Rosen, D. L. (1985). Use of product-testing organizations' recommendations as a strategy for choice simplification. Journal of Consumer Affairs, 19(1), 118-139.

Palacio, A. B., Meneses, G. D., \& Pérez, P. J. P. (2002). The configuration of the university image and its relationship with the satisfaction of students. Journal of Educational Administration, 4O(5), 486-505.

Parasuraman, A., \& Grewal, D. (2000). The impact of technology on the quality-value-loyalty chain: A research agenda. Journal of the Academy of Marketing Science, (28), 168-174.

Pate, W. S. (1990). Modeling consumer satisfaction, determinants of satisfaction, and post-purchase actions among, consumers of undergraduate higher education (Unpublished doctoral dissertation). University of North Carolina, Chapel Hill.

Petrick, J. F. (2002). Development of a multi-dimensional scale for measuring the perceived value of a service. Journal of Leisure Marketing, 34(2), 119-134.

Petrick, J. F. (2004). The roles of quality, value, and satisfaction in predicting cruise passengers' behavioral intentions. Journal of Travel Research, 42, 397-407.

Ping, C. (1993). Accountability in higher education. The Ohio CPA Journal, 11-18.

Rosa, M. J., Saraiva, P., \& Diz, H. (2000). Um modelo de excelência para instituições de ensino superior. Anais do Congresso Nacional da Qualidade, Lisboa, 1, 1-7. 
Sander, P., Stevenson, K., King, M., \& Coates, D. (2000). University students' expectations of teaching. Studies in Higher Education, 25(3), 309-323.

Santos, P. H. (2016). Proposição e teste da escala ECSI adaptada para avaliação da qualidade no ensino superior (Dissertação de Mestrado, Centro Universitário UNA, Belo Horizonte, Minas Gerais, Brasil).

Schumacker, R. E., \& Lomax, R. G. (2004). A beginner's guide to structural equation modeling (2nd ed.). Lawrence Erlbaum Associates Publishers.

Sheth, J., Newman, B. I., \& Gross, B. L. (1991). Consumption values and market choice: Theory and applications. South Western.

Sun, K., \& Kim, D. (2013). Does customer satisfaction increase firm performance? An application of American Customer Satisfaction Index (ACSI). International Journal of Hospitality Management, 35, 68-77.

Thompson, B. (2004). Exploratory and confirmatory factor analysis: Understanding concepts and applications. American Psychological Association.

Tomio, J. L., \& Souza, M. J. B. (2008). Satisfação dos alunos de administração com o curso e sua relação com a imagem e satisfação da IES. Revista de Negócios, 13(2), 105-121.

Turkyilmaz, A., Temizer, L., \& Oztekin, A. (2018). A causal analytic approach to student satisfaction index modeling. Annals of Operations Research, 263(1-2), 565-585.

Wilkie, W. L. (1994). Consumer behavior. John Wiley \& Sons.

Wilkins, S., \& Balakrishnan, M. S. (2013). Assessing student satisfaction in transnational higher education. International Journal of Educational Management.

Zeithaml, V. (1987). Defining and relating price, perceived quality and perceived value. Cambridge: MSI.

Zink, K., \& Vob, W. (1999, August 30-31). The new EFQM excellence model and its impact on higher education institutions. Proceedings of the TQM for Higher Education Institutions Conference: Higher Education Institutions and the Issue of Total Quality, Verona, 241-255. 